Scientific Visualization, 2020, volume 12, number 2, pages 127 - 138, DOI: 10.26583/sv.12.2.10

\title{
Visualization of bibliometric networks of scientific publications on the study of the human factor in the operation of nuclear power plants based on the bibliographic database Dimensions
}

\author{
A.Kh. Khakimova ${ }^{1, A}$, O.V. Zolotarev²,B, M.A. Berberova3,A,B,C \\ A Autonomous non-profit organization "Scientific and Research Center for Information in \\ Physics and Technique" \\ B Autonomous non-profit organization of higher education "Russian New University" \\ c Autonomous non-profit organization "International Nuclear Safety Center" \\ ${ }^{1}$ ORCID: 0ooo-0oo1-9355-9249, aida khatif@mail.ru \\ 2 ORCID: 00oo-0oo1-6917-9668,ol-zolot@yandex.ru \\ 3 ORCID: 0000-0002-6357-7929, maria.berberova@gmail.com
}

\begin{abstract}
The reliability of the human operator is an essential indicator of the safe operation of nuclear power plants. Mistakes can be made during performance checks, maintenance, at the stage of accident management, etc.

The need to take into account the human factor in the analysis of safety and reliability of nuclear power plants is justified by the fact that erroneous actions of personnel and operators of nuclear power plants directly or indirectly can lead to accidents. Therefore, the analysis of personnel reliability (ANP) when performing a probabilistic safety analysis (PSA) and risk assessments afford to identify the most likely erroneous actions of NPP personnel and to develop a set of measures to reduce them.

Depending on the likely consequences of the accident and the time available to the operator for the intervention, the personnel will be exposed to various levels of stress, which will directly affect the personnel's actions to troubleshoot in the event of an emergency. If an accident has occurred, then in a short period of time the operator needs to make a number of decisions, the correctness of which can both save and aggravate the situation.

This paper considers the issues of visualization of bibliometric networks of scientific publications on the study of the human factor in the operation of nuclear power plants.
\end{abstract}

Keywords: bibliometric analysis, visualization, citation network.

\section{Introduction}

The reliability of the human operator is an essential indicator of the safe operation of nuclear power plants. Mistakes can be made during performance checks, maintenance, at the stage of accident management, etc. The stability of a nuclear power plant is affected by a number of different factors:

- level of organization of the project;

- quality of equipment in operation;

- selection and training of competent personnel;

- maintaining the qualifications of NPP workers

and etc.

In the "man-machine" system, the reliability of the technical component is calculated by known methods and in accordance with established reliability standards. However, the "hu- 
man" component cannot be technically accurately determined, therefore, it is necessary to undertake systematic efforts to increase and subsequently maintain the achieved level of reliability of this component.

The most significant contribution to emergency situations at hazardous facilities is made by personnel. $70 \%$ of disasters are caused by improper actions or low personnel reliability, and only 30\% are equipment failure, adverse effects of external factors, etc.

\section{Methods and materials}

The Dimensions Database (http://app.dimensions.ai.) is an advanced scientific database that includes not only books, chapters, and conference materials, but also provides grants, patents, clinical trials, program documents, and altmetric information.

The metadata for this database was obtained from sources such as CrossRef, PubMed, Directory of Open Access Journals, Open Citation Data, clinical trial registries, patent offices and more than 100 publishers [1]. In some cases, full-text data is indexed from open sources, such as PubMed Central and arXiv, providing greater detection and access capabilities than other citation databases [2].

Launched in January 2018, Dimensions brings together more than 133 million research documents throughout the research life cycle. The free version of the platform provides open access to more than 95 million publication records and related metrics.

Citation counting is the basis of bibliometric analysis since the late 1950 os and an assessment tool from the mid-1980s. Dimensions builds its citation graph using several methods [3].

Identification of the organization, disambiguation of authors, processing in a natural language, and extraction of links mean that Dimensions can accurately respond to complex search queries [4].

VOSviewer is a software tool that is fully focused on the visualization of bibliometric networks. In the visualizations provided by VOSviewer, the distance between the two nodes indicates their relationship. Thus, VOSviewer is especially suitable for visualizing of large networks.

In a bibliometric network, there are often large differences between nodes in terms of the number of edges they have. Popular sites, for example, representing highly cited publications, can have several orders of magnitude more links than their less popular counterparts. When analyzing bibliometric networks, normalization of these differences between nodes is usually performed. VOSviewer by default applies the normalization of the strength of communication [5].

The next step after building a normalized network is to place the nodes in the network in twodimensional space so that the strongly connected nodes are close to each other, while the weakly connected nodes are far from each other. For this purpose, the VOS matching technique is used (VOS - "visualization of similarities") [6].

In addition, VOSviewer by default assigns nodes in the network to clusters. A cluster is a collection of closely related nodes. Each node in the network is assigned to only one cluster. The number of clusters is determined by the resolution parameter. To indicate the cluster assigned to this node, VOSviewer (when visualizing the bibliometric network) uses colors [7].

In addition, VOSviewer supports overlay renderings. In overlay rendering, the color of a node indicates a specific property of the node. For example, nodes can represent magazines, and the color of a node can indicate the number of links to a given journal [8]. Another visualization supported by VOSviewer is density visualization [9].

The search was carried out on the Dimensions platform for the keywords "nuclear power plants human factor" in the names and annotations for all years. 


\section{Results}

746 publications were found in the Dimensions database. There are of them 520 articles, 125 abstracts, 94 chapters from books, 5 monographs, 2 preprints.

The publication activity in the field of human factor research in the operation of nuclear power plants is shown in fig. 1 (including all types of publications). The trend line is exponential, the coefficient of determination (R2), also called the "approximation confidence value", is 0.6719 .

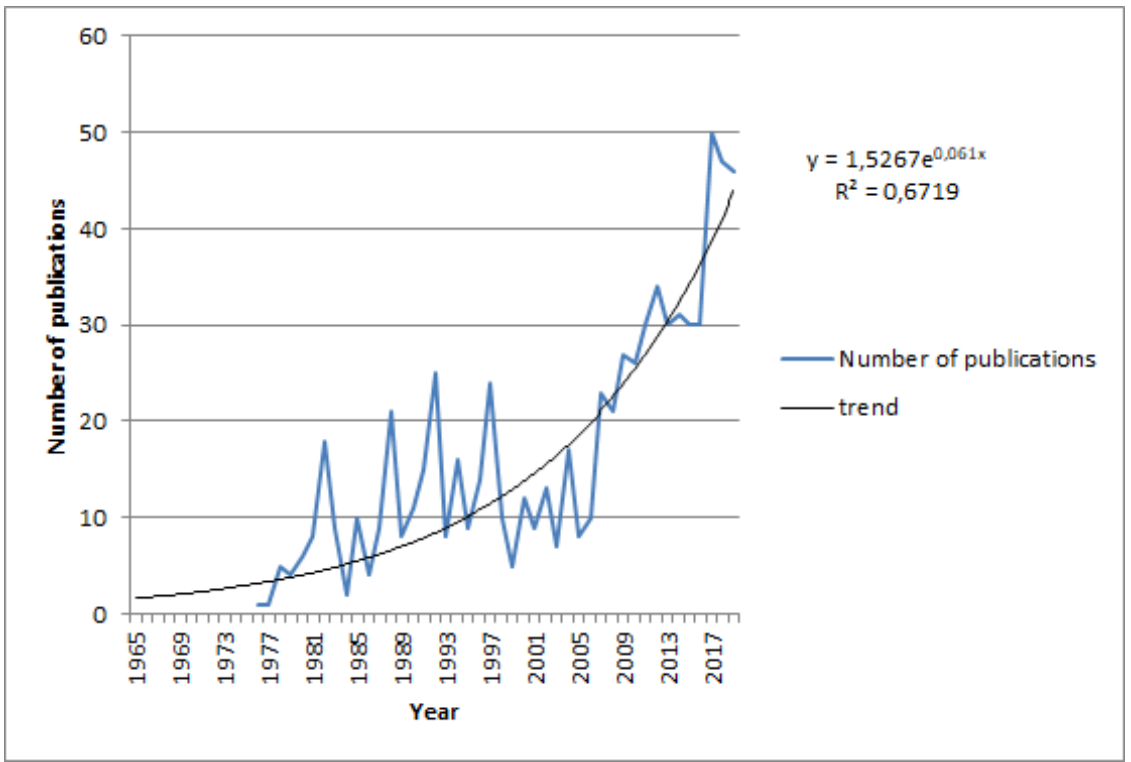

Fig. 1. Publication activity in the field of human factor research in the operation of nuclear power plants based on the bibliographic database Dimensions.

The top 20 countries with the largest number of publications (Fig. 2) include such countries as the USA (162 articles), South Korea (65), China (62), Japan (32), Taiwan (18), and Great Britain (14), France (12), Brazil, Finland, India (10 articles each). Russia is in 16th place with 7 jobs.

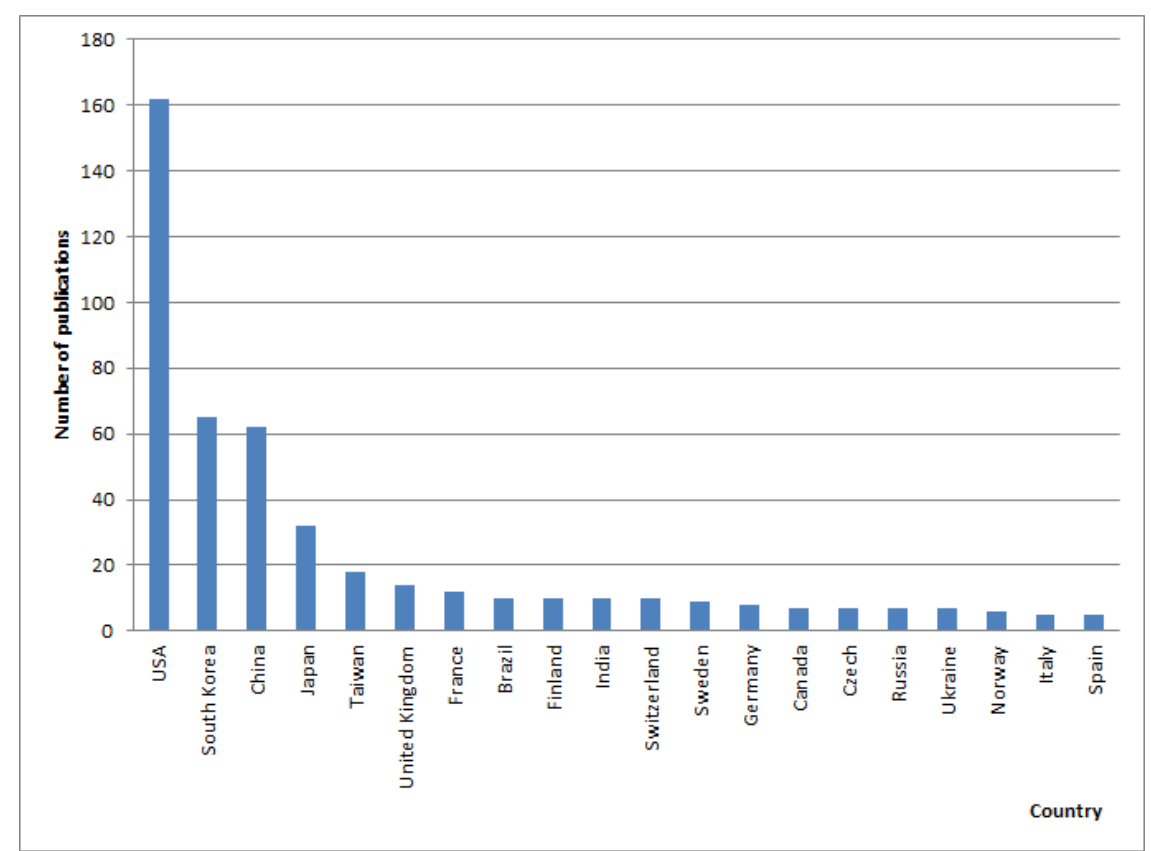

Fig. 2. Top 20 countries with the most number of publications. 
When building a co-authorship network for 1665 authors from 43 countries, the minimum number of articles by the author was taken to be five. There were 36 such authors in 11 clusters (Fig. 3). The largest clusters included 8 authors (lead author is Seong Poong Hyun), 8 (lead author is Boring Ronald L.) and 5 authors (lead author is Zhang Li). The smallest clusters included one author. The network shows a high degree of separation of authors from different countries, and even within the same country (for example, "Chinese" clusters - 6).

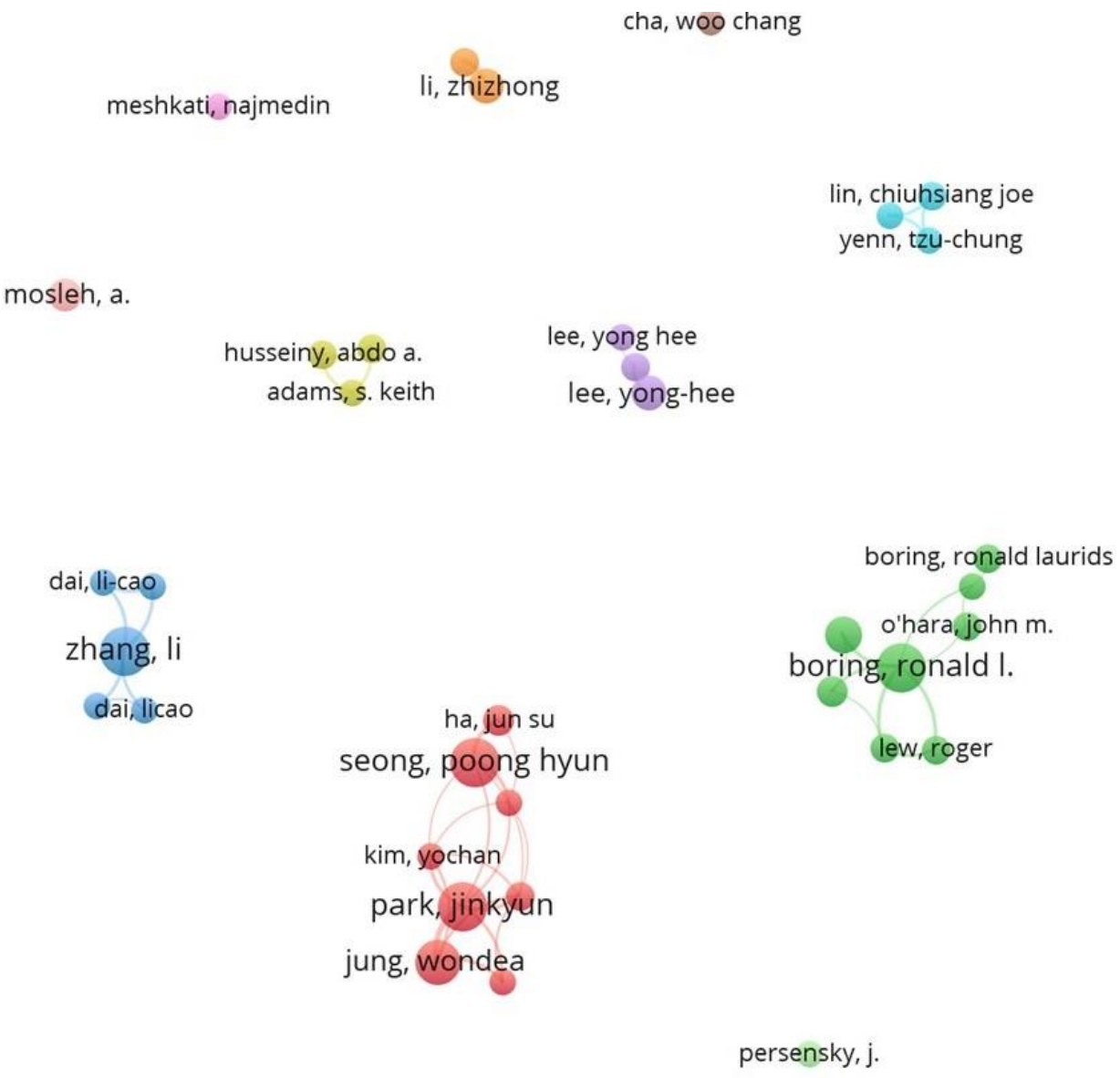

Fig. 3. Co-authoring visualization of a network of authors.

A network of scientists based on citation was built. In this case, the connection between the researchers will be formed when citing them in the same sources. The size of the node in the network depends on the number of links to this particular researcher.

The minimum number of citations was taken to be 20 . At the same time, 40 authors were identified (Fig. 4). 


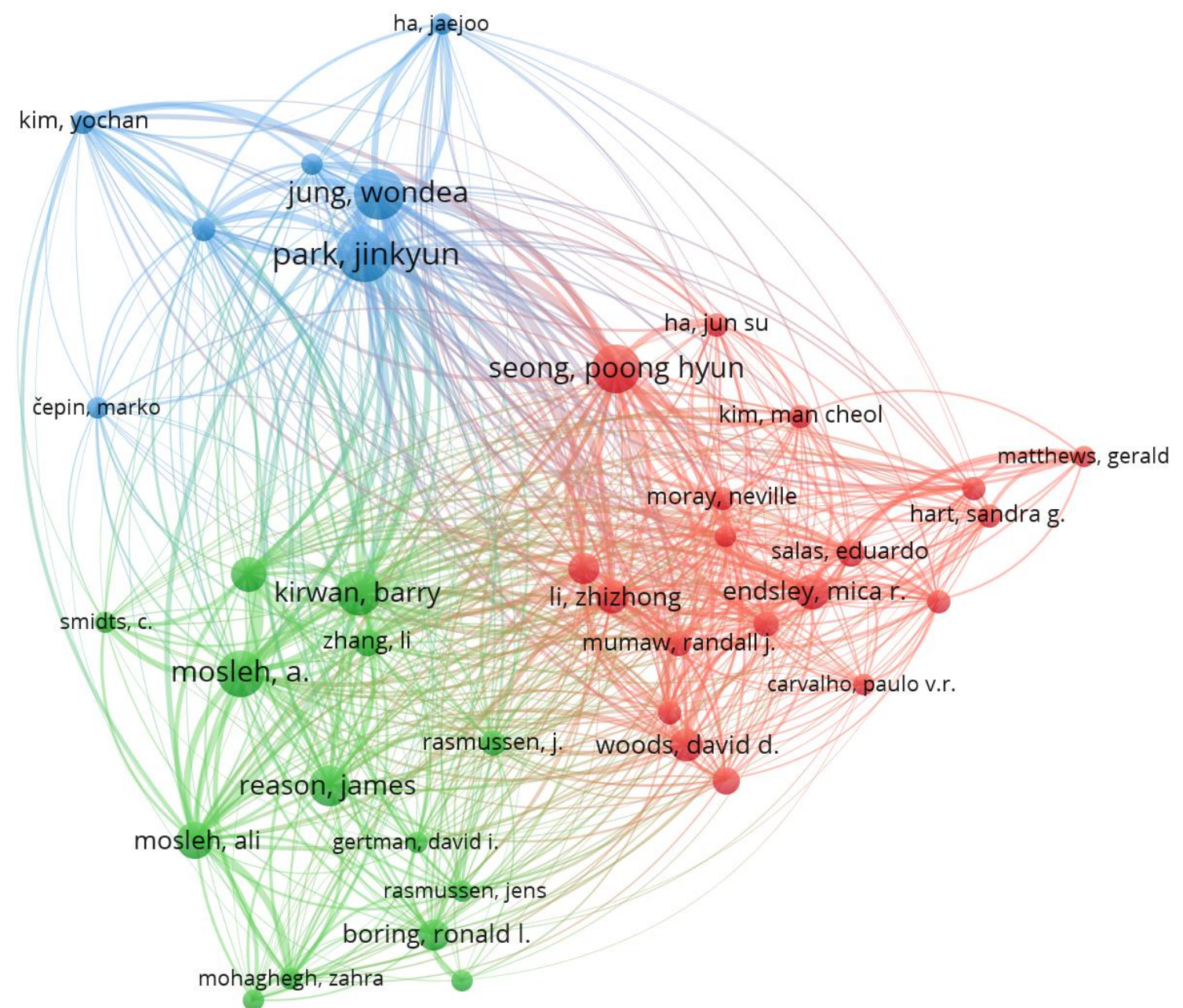

Fig. 4. Visualization of a network of authors based on citation.

3 international clusters of authors were identified, including 19, 14 and 7 authors, respectively. The central author of the 1st cluster is Seong Poong Hyun, for the 2nd cluster - Park Jinkuyn, for the 3 rd cluster - Mosleh A. Therefore, scientists are familiar with the works of authors of other research groups.

A network of scientists was built on the basis of a bibliographic combination. In the case of a bibliographic combination, a link between the researchers will be built if they quote the same sources. The size of the network node depends on how the researcher quoted other scientists. The minimum number of citations was taken equal to 5, 36 authors were identified (Fig. 5). 


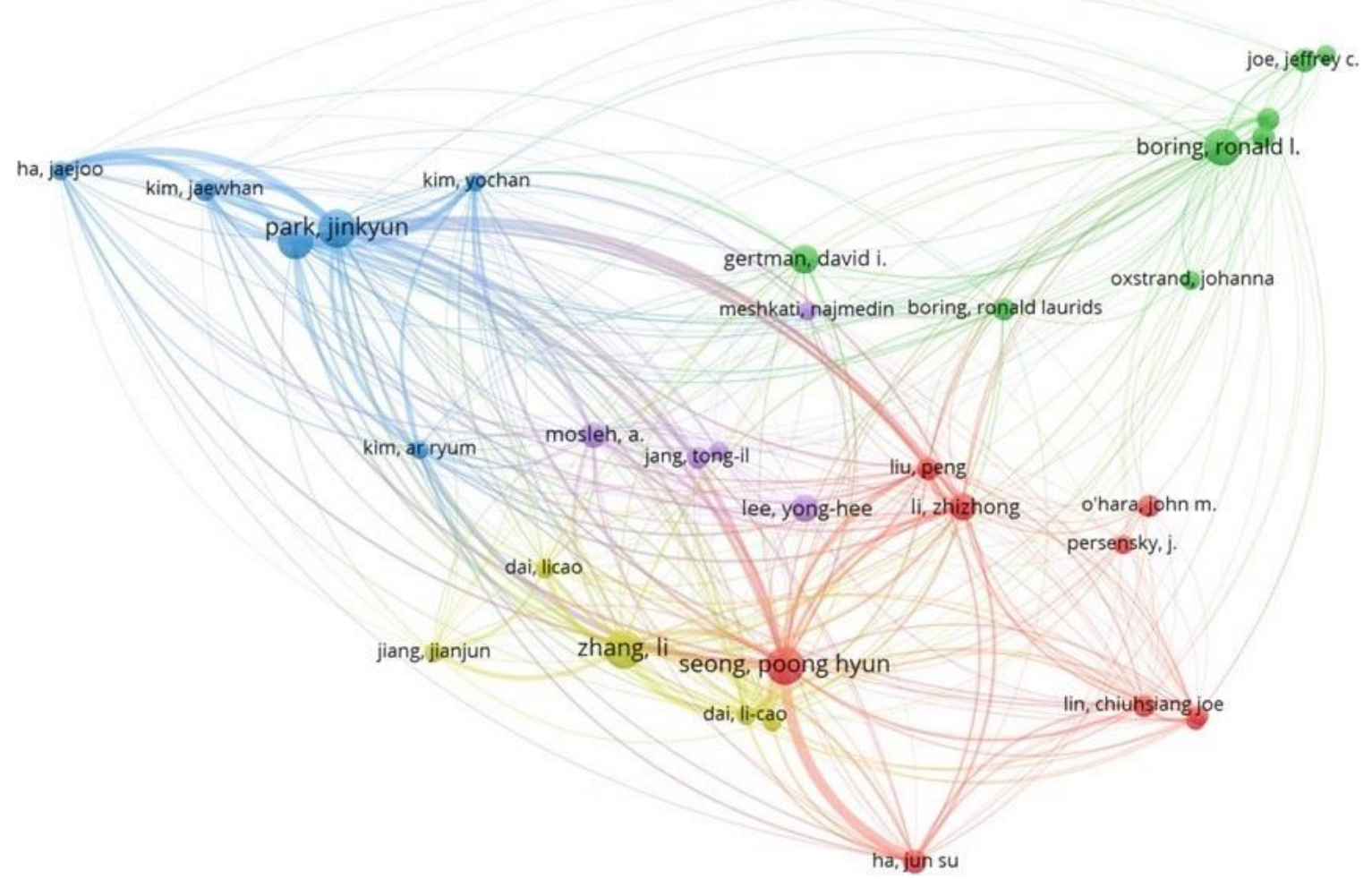

Fig. 5. Network of authors based on bibliographic combination

Four clusters were identified, including Chinese authors (the central authors are Park Jinkuyn, Seong Poong Hyun, Zhang Li). The central author of the international cluster is Boring Ronald.

Therefore, in both co-authoring and citation and bibliographic combination networks, the central authors are a small list of scientists, including Park Jinkuyn, Seong Poong Hyun, Zhang Li, Boring Ronald.

In order to identify the most influential organizations for the study of the human factor in the management of nuclear power plants, visualization of co-authorship networks by organizations was performed. The minimum number of articles of the organization was taken equal to three. Out of 297 organizations, 52 organizations were selected that met these requirements (Fig. 6). 
china general nuclear power corporation (china)

iowa state university

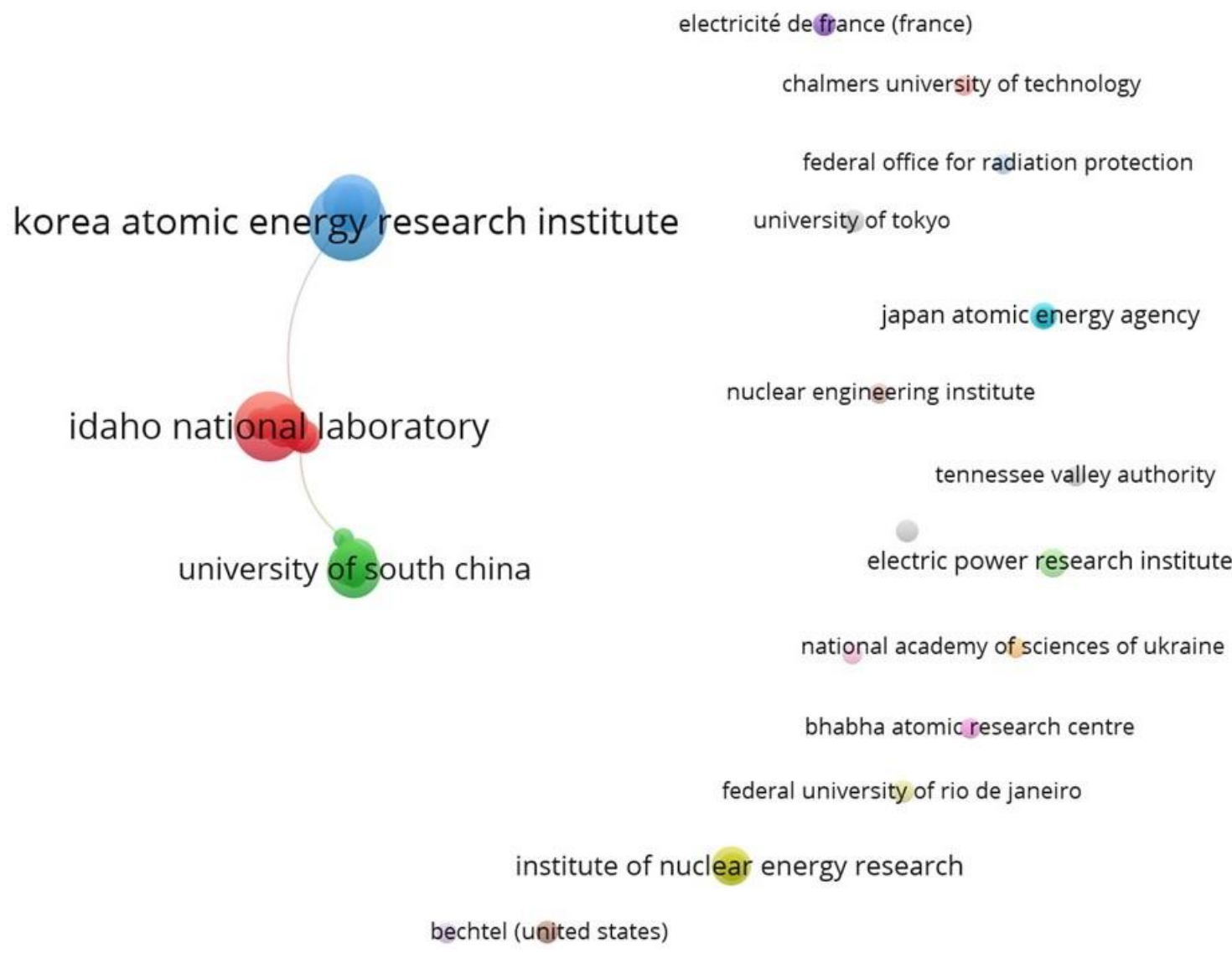

Fig. 6. Organization Collaboration Network Visualization

The visualization of a collaborative network of organizations confirms the fragmentation of research. Only three organizations have common publications; these are the University of South China (China), the National Idaho Laboratory (USA), and the Korean Atomic Energy Research Institute (South Korea). The remaining organizations conduct independent research, including the National Academy of Sciences of Ukraine.

An analysis of co-authorship by country was carried out, the minimum number of articles of the country was taken to be three. Of the 43 countries, 26 were allocated. These 26 countries made up two clusters (Fig. 7). The 1st cluster includes 16 countries: Austria, Canada, China, Finland, France, Germany, India, Italy, Norway, Russia, South Korea, Sweden, Switzerland, Turkey, United Arab Emirates, Great Britain. The central country is South Korea. The 2nd cluster includes 7 countries: Australia, Brazil, Czech Republic, Japan, Taiwan, Ukraine, and the USA. The central country is the USA.

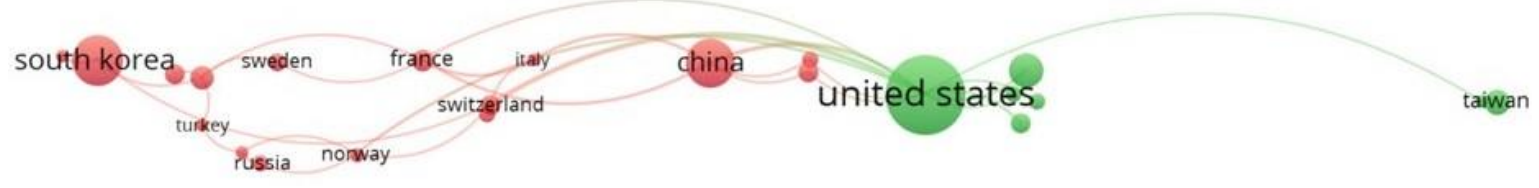

Fig. 7. Country Collaboration Network Visualization

A citation analysis was carried out for publication sources, the minimum number of publications in the source was taken to be five. The 23 most cited sources were identified (Fig. 8). 
The most cited sources are Reliability Engineering \& System Safety, Annals of Nuclear Energy, Proceedings of the Human Factors and Ergonomics Society Annual Meeting.

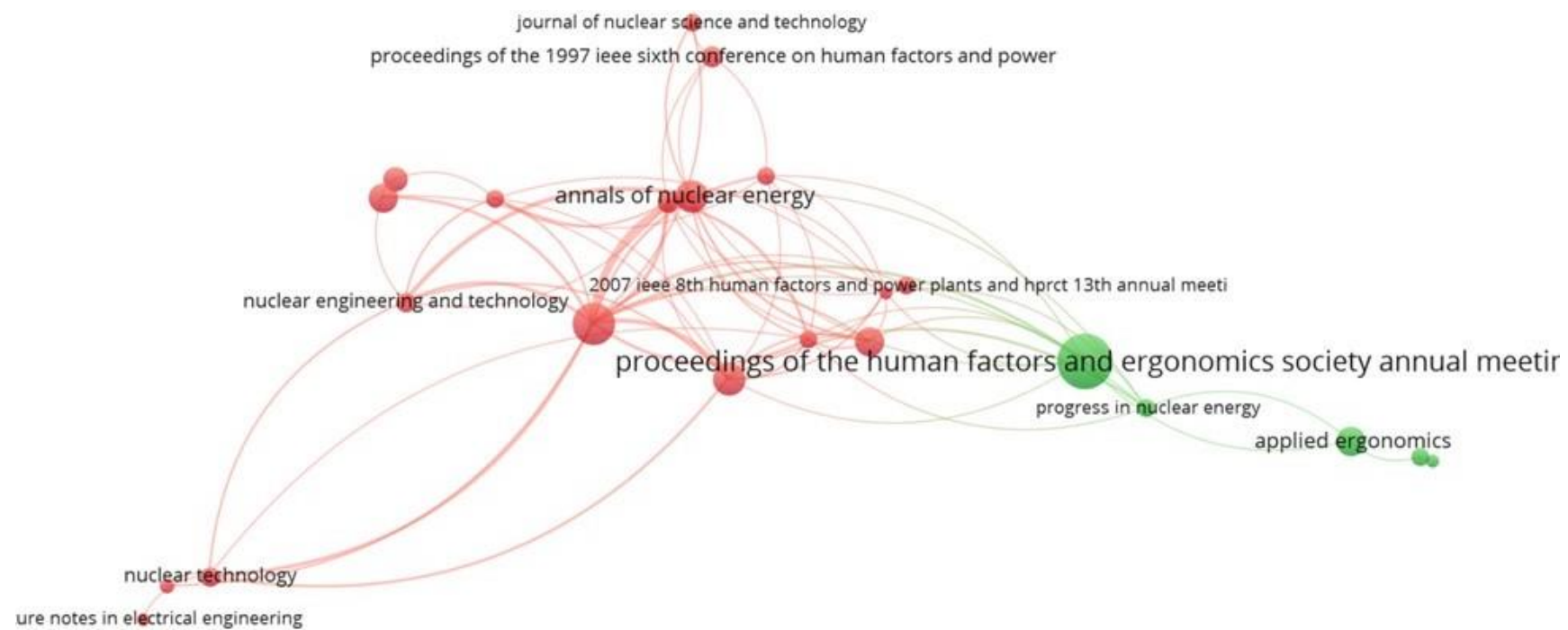

Fig. 8. Citation Network Visualization by Source

We reviewed a citation network by country. The minimum number of publications in the country was taken to be five (Fig. 9). 19 countries were identified in 3 clusters. The 1st cluster includes 8 countries: China, Czech Republic, Germany, India, Italy, Japan, South Korea, and Great Britain. The second cluster included 6 countries: Brazil, France, Norway, Russia, Sweden, Taiwan. Cluster 3 includes 5 countries: Canada, Finland, Spain, Switzerland, and the USA.

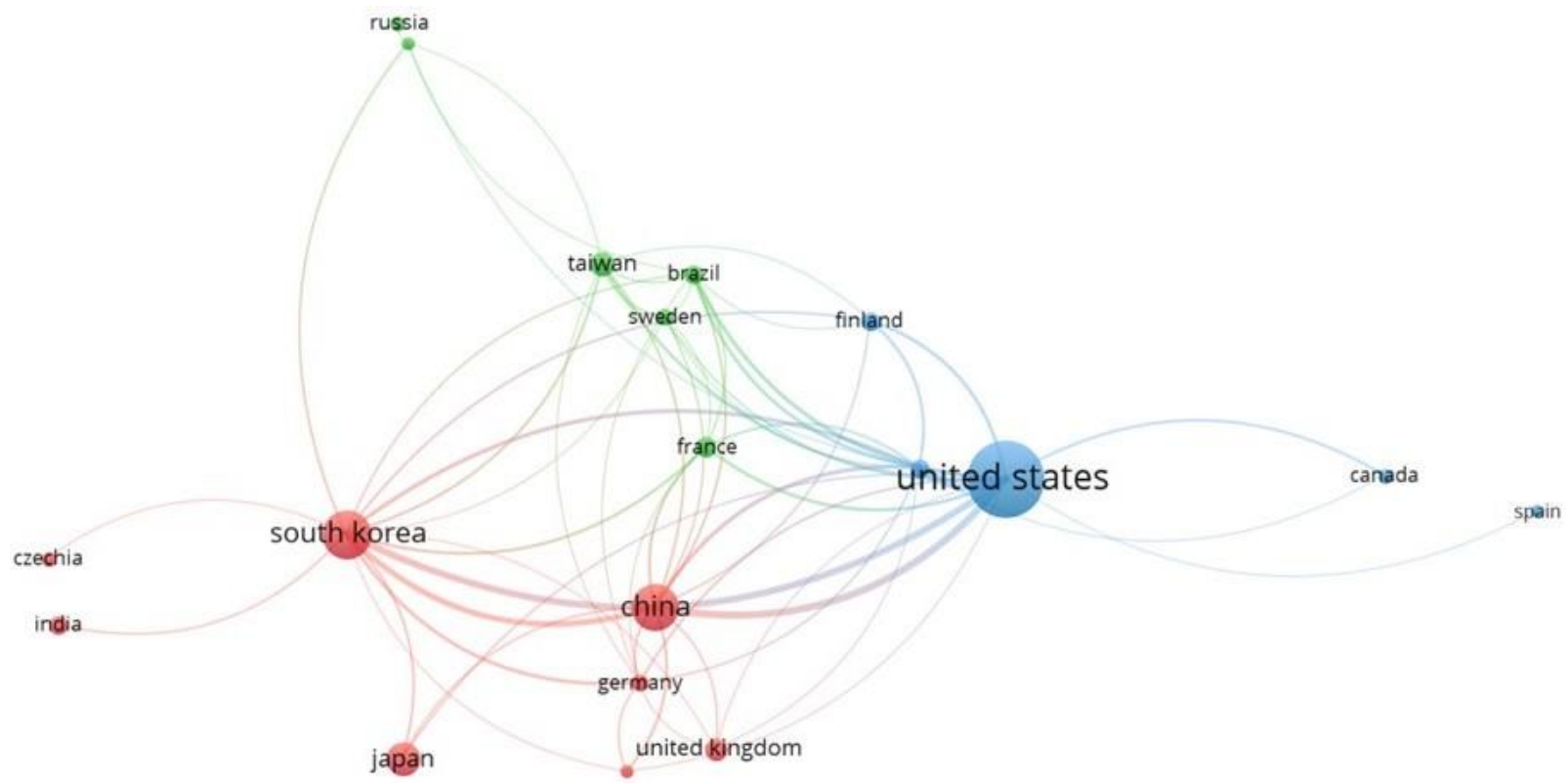

Fig. 9. Country Citation Network Visualization

We looked at a citation network for organizations. The minimum number of publications in the country was taken to be five (Fig. 10). 24 organizations were identified, the authors of which cite each other.

The first cluster includes 11 organizations: 
- Chosun University, Korea Advanced Institute of Science and Technology, Korea Atomic Energy Research Institute, Korea Institute of Nuclear Safety (South Korea);

- China General Nuclear Power Corporation, Harbin Engineering University (China), Institute of Nuclear Energy Research, Chung Yuan Christian University (Taiwan);

- Electric Power Research Institute, United States Nuclear Regulatory Commission (USA);

- Institute for Energy Technology (Norway).

- The second cluster includes 7 organizations:

- Hunan Institute of Technology, Nuclear and Radiation Safety Center, College Park, University of South China (China);

- the Ohio State University, University of Maryland, Sandia National Laboratories (USA);

- Paul Scherrer Institute (Switzerland).

- The third cluster includes 6 organizations:

- Brookhaven National Laboratory, Idaho National Laboratory, University of Idaho, (USA);

- National Tsing Hua University (Taiwan), Tsinghua University (China);

- Vtt Technical Research Center of Finland (Finland).

Therefore, three groups of collaborating organizations can be distinguished. The first group is headed by South Korean institutions. At the head of the second group - Chinese, at the head of the third group - American.

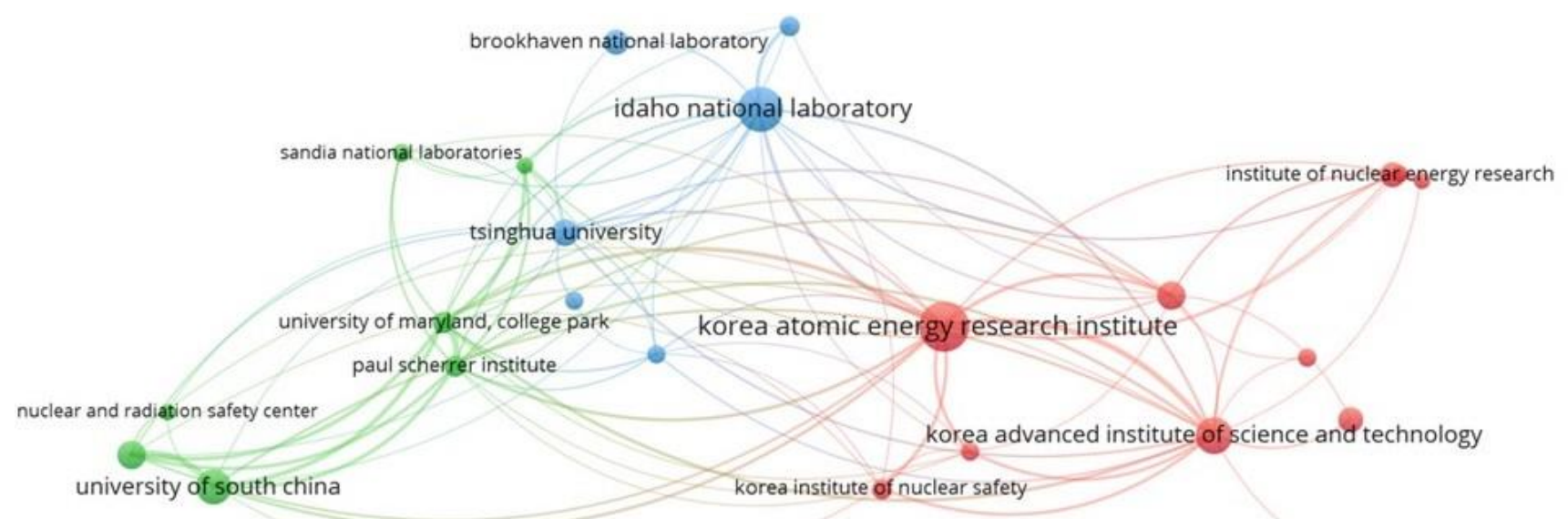

Fig. 10. Organization Citation Network Visualization

More than one and a half thousand authors writing in English work in the subject under study. The visualization results show the authors that do not participate in large international collaborations. VOSviewer features "isolated" research teams and collaboration between individual authors.

To identify the most cited articles, we built a network of visualization of the most cited documents, taking the minimum number of citations equal to fifteen. 39 articles were identified in five clusters (Fig. 11). 


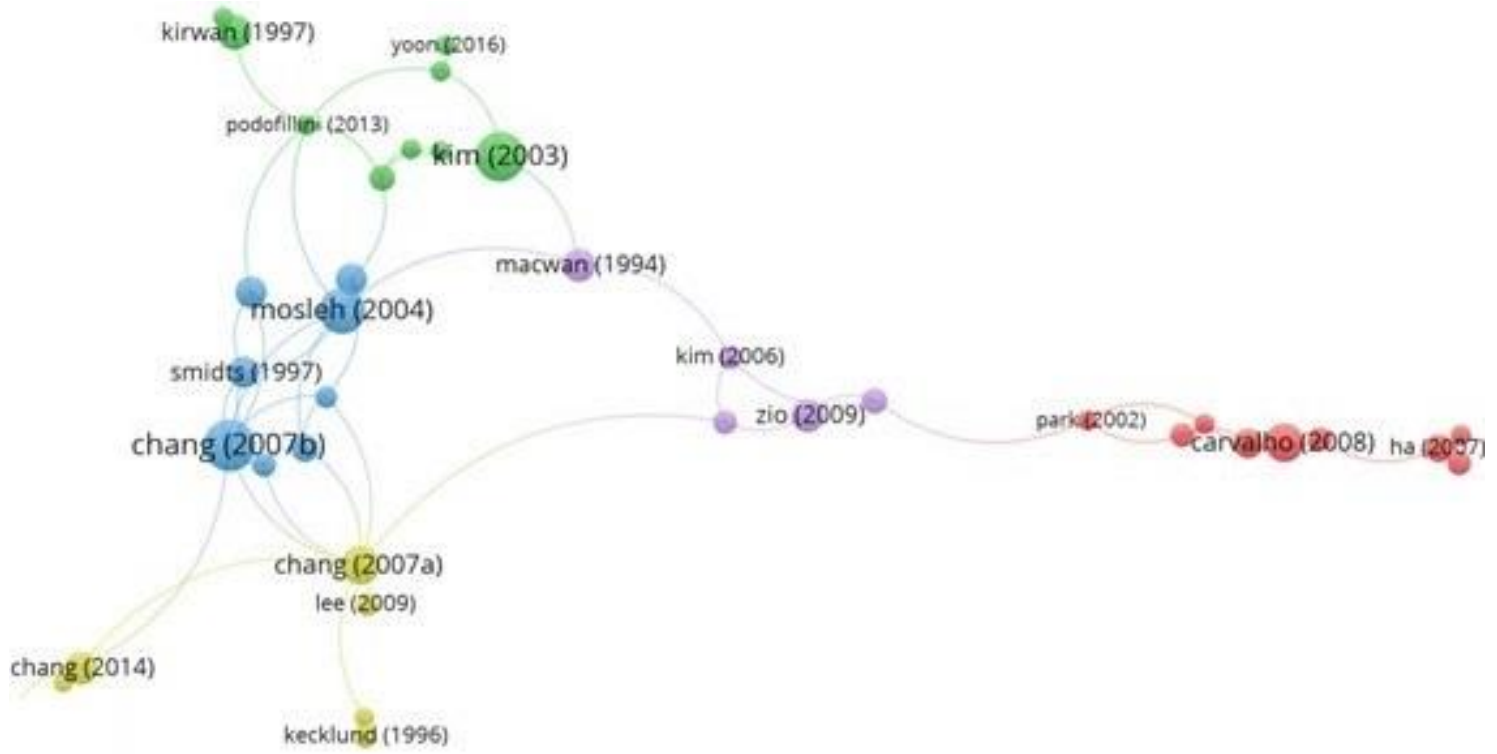

Fig. 11. Visualization of the networks of the most cited documents

The first cluster (red, 9 articles) is formed around the article "Human Factors Approach for Evaluation and Redesign of Human - System Interfaces of a Nuclear Power Plant Simulator" (2008) [10].

The second cluster (green, 9 articles) is grouped around the article "A Taxonomy of Performance Influencing Factors for Human Reliability Analysis of Emergency Tasks" (2003) [11].

The third cluster (blue, 8 articles) is formed around the publication "Cognitive Modeling and Dynamic Probabilistic Simulation of Operating Crew Response to Complex System Accidents. Part 1: overview of the IDAC model" (2007) [12].

A fourth cluster (olive) is formed around the article "Cognitive Modeling and Dynamic Probabilistic Simulation of Operating Crew Response to Complex System Accidents. Part 2: IDAC Performance Influencing Factors Model" (2007) [13].

The fifth cluster (purple) is grouped around the article "a fuzzy set-based approach for modeling dependence among human errors" (2009) [14].

In accordance with central publications, we can identify the following relevant areas of research development:

1) the consideration of the human factor in the assessment and redesign of man-system interfaces of nuclear power plants using simulators as examples;

2) the taxonomy of factors affecting productivity, for the analysis of human reliability in emergency situations;

3) cognitive modeling and dynamic probabilistic modeling of the response of the working team to complex systemic accidents using the IDAC model as an example;

4) modeling the relationship between human errors based on a fuzzy set-based approach.

IDAC is a model of information, decisions and actions in the context of the crew for analyzing human reliability. The model is designed for probabilistic forecasting of the response of the team managing the NPP dispatcher during an accident for use in probabilistic risk assessments. The response spectrum of the operator includes cognitive, emotional and physical actions during the accident. IDAC includes a crew model of three types of operators [12, 13]. This work was supported by the Russian Foundation for Basic Research, grants No. 17-0701475, 18-07-00225, 18-07-00909, 18-07-01111 and 19-07-00455. 


\section{Referencies}

1. Hook DW, Porter SJ, Herzog C. Dimensions: building context for search and evaluation. Front Res Metr Anal. 2018 Aug 23; 3: 23. doi: 10.3389 / frma.2018.00023. doi: 10.3389 / frma.2018.00023. [

2. Schonfeld R. A new citation database launches today: Digital Science's Dimensions. Scholarly Kitchen [Internet]. $\quad$ Jan $15, \quad 2018$. https://scholarlykitchen.sspnet.org/2018/01/15/new-citation-database-dimensions.

3. Hook D.W., Porter S.J. and Herzog C. Dimensions: Building Context for Search and Evaluation. Front Res. Metr. Anal., 2018. 3:23. doi: 10.3389 / frma.2018.00023

4. Williams C. Dimensions from Digital Science, 2018. Insights 31: 33. DOI: http://doi.org/10.1629/uksg. 420

5. Van Eck, N.J., \& Waltman, L. How to normalize cooccurrence data? An analysis of some well-known similarity measures. Journal of the American Society for Information Science and Technology, 2009. 60 (8), 1635-1651.

6. Van Eck, N.J., Waltman, L., Dekker, R., \& Van den Berg, J. A comparison of two techniques for bibliometric mapping: Multidimensional scaling and VOS. Journal of the American Society for Information Science and Technology, 2010. 61 (12), 2405-2416.

7. Waltman, L., Van Eck, N.J., \& Noyons, E.C.M. A unified approach to mapping and clustering of bibliometric networks. Journal of Informetrics, 2010. (4), 629-635.

8. Van Eck, N.J., Waltman, L., Van Raan, A.F.J., Klautz, R.J.M., \& Peul, W.C. Citation analysis may severely underestimate the impact of clinical research as compared to basic research. PLoS ONE, 2013.8 (4), e62395.

9. Van Eck, N.J., \& Waltman, L. (2014). Visualizing bibliometric networks. In Y. Ding, R. Rousseau, \& D. Wolfram (Eds.), Measuring scholarly impact: Methods and practice. 2014 (pp. 285-320). Springer

10. Carvalho P.V.R., Dos Santos I.L., Gomes J.O., Borges M.R.S., Guerlain S. Human Factors Approach for Evaluation and Redesign of Human - System Interfaces of a Nuclear Power Plant Simulator. Displays, 2008.29 (3), 273-284.

11. Kim J.W., Jung W. A Taxonomy of Performance Influencing Factors for Human Reliability Analysis of Emergency Tasks. Journal of Loss Prevention in the Process Industries, $2003.16(6), 479-495$.

12. Chang Y.H.J., Mosleh A. Cognitive Modeling and Dynamic Probabilistic Simulation of Operating Crew Response to Complex System Accidents. Part 1: overview of the IDAC model. Reliability Engineering \& System Safety, 2007. 92 (8), 997-1113.

13. Chang Y.H.J., Mosleh A. Cognitive Modeling and Dynamic Probabilistic Simulation of Operating Crew Response to Complex System Accidents. Part 2: IDAC Performance Influencing Factors Model». Reliability Engineering \& System Safety, 2007. 92(8), 10141040.

14. Zio E., Baraldi P., Librizzi M., Podofillini L., Dang V.N. A Fuzzy Set-Based Approach for Modeling Dependence Among Human Errors. Fuzzy Sets and Systems, 2009. 160(13), 1947-1964. 